$\mathrm{R} / 95 / 12$

hep-th/9504095

April, revised May, 1995

\title{
String-Membrane Duality in Seven Dimensions
}

\author{
P. K. TOWNSEnd \\ DAMTP, University of Cambridge, \\ Silver Street, Cambridge CB3 9EW, U.K.
}

\begin{abstract}
The conjectured equivalence of the heterotic string to a $K_{3}$ compactified type IIA superstring is combined with the conjectured equivalence of the latter to a compactified 11-dimensional supermembrane to derive a string membrane duality in seven dimensions; the membrane is a soliton of the string theory and vice versa. A prediction of this duality is that the heterotic string is a $K_{3}$ compactification of the solitonic 11-dimensional fivebrane. It is verified that the worldsheet action of the $\mathrm{D}=10$ heterotic string is indeed obtainable by $K_{3}$ compactification of the worldvolume action of the 11-dimensional fivebrane, and it is suggested how the worldvolume action of the $\mathrm{D}=11$ supermebrane may be similarly obtained by $T^{3}$ compactification of the worldvolume action of a $\mathrm{D}=10$ heterotic fivebrane. Generalizations to $D=8$ string-threebrane and membrane-membrane duality are also discussed.
\end{abstract}




\section{Introduction}

According to a recent proposal [1], the ten-dimensional $(\mathrm{D}=10)$ type IIA superstring theory is an $S^{1}$ compactification of the, still conjectural, 11-dimensional $(\mathrm{D}=11)$ supermembrane theory [2]. The impetus for this proposal came from the realization that, in the quantum theory, the extreme electric black holes of the $\mathrm{D}=10 \mathrm{~N}=2 \mathrm{~A}$ supergravity theory [3] would be indistinguishable from a tower of Kaluza-Klein (KK) states. This observation has since been made independently by Witten [4] who has also pointed out that since the $S^{1}$ radius goes to infinity with the string coupling constant, the strong coupling limit of the type IIA superstring theory can be viewed as a decompactification limit in which 11-dimensional Lorentz invariance is restored. These observations are a clear indication that the type IIA superstring theory has an 11-dimensional origin, but they do not, by themselves, provide evidence for an 11-dimensional supermembrane. Indeed, the only low mass excitations of the type IIA superstring in the strong coupling limit are the massless excitations of 11-dimensional supergravity [4]. The reason for the identification in [1] of the 11-dimensional theory as a supermembrane theory, rather than merely $\mathrm{D}=11$ supergravity, is to be found partly in earlier work identifying the type IIA worldsheet action as a double dimensional reduction of the supermembrane worldvolume action [5], also partly in work [6,7,8] relating the 'solitonic' string [9] and other p-brane solutions [3] of $\mathrm{D}=10 \mathrm{~N}=2 \mathrm{~A}$ supergravity to the membrane [6] and fivebrane [10] solutions of 11-dimensional supergravity, and in [11] where it was suggested, on the basis of U-duality in type II string theory, that the 'solitonic' membrane of $\mathrm{D}=11$ supergravity be identified with a fundamental supermembrane.

This proposal is consistent with the observations of [4] concerning the low mass excitations in the strong coupling limit of the type IIA superstring if (i) the zero mass excitations of the supermembrane are those of $D=11$ supergravity, as advocated in [1] partly on the basis of earlier work $[12,13]$ on supermembranes *, and (ii) there are no other low mass excitations, which would not be surprising

\footnotetext{
$\star$ But see [14] for a review of the arguments against.
} 
in view of the absence in 11 dimensions of a dilaton field, and hence of any weak coupling limit.

According to another proposal [11], the $K_{3}$-compactified type IIA superstring is equivalent, non-perturbatively, to a toroidally compactified heterotic string. The basis for this proposal was that (i) the low energy effective supergravity theories are the same and (ii) the spectrum of massive 'Bogomolnyi' states may also be the same once one includes 'wrapping' modes of solitonic p-brane solutions of the ten-dimensional theories; these are analogous to string winding states, but are non-perturbative. Thus, while the perturbative spectrum of massive states is very different in the two theories, this difference could disappear in the full theory. Whether this actually happens ${ }^{\dagger}$ depends on a number of factors, one of which is whether the $\mathrm{D}=10 \mathrm{p}$-brane solutions in question are truly 'solitonic' and have therefore to be included when determining the spectrum of a compactified string theory. If 'solitonic' is taken to mean that the 'string sigma-model' spacetime metric is geodesically complete and that all fields are everywhere non-singular then, of the type IIA p-brane solutions, only the neutral fivebrane $[16,17]$ is truly 'solitonic'. For the heterotic string theory this suffices since the only other pbrane solution is the 'solitonic' string and while this is not actually solitonic by the above criterion ${ }^{\ddagger}$, this fact is unimportant if this solution is identified with the fundamental string, as suggested in $[18,11]$. For the type IIA superstring, a similar justification of the necessity of including p-brane solitons is possible only if they are re-interpreted as solutions of $\mathrm{D}=11$ supergravity and then only if the membrane solution is identified with a fundamental supermembrane because, unlike the $\mathrm{D}=11$ fivebrane, the $\mathrm{D}=11$ membrane solution has a (timelike) singularity behind its horizon; this fact was one of the principal motivations of [1]. Thus the conjectured equivalence of the heterotic string with the $K_{3}$ compactification of the type IIA superstring is not independent of the identification of the latter

\footnotetext{
$\dagger$ And evidence that it does is accumulating $[4,15]$.

$\ddagger$ This is equally true in terms of the 'fivebrane sigma-model' metric because the dilaton blows up at finite affine parameter along timelike geodesics.
} 
as a compactified supermembrane. It is therefore natural to consider these two conjectures in conjunction with each other. This has already been done in [4] to the extent that the strong coupling dynamics of the $\mathrm{D}=7$ heterotic string was related to a $K_{3}$ compactification of 11-dimensional supergravity. The purpose of this paper is to consider the implications of supposing the strong coupling limit of the $\mathrm{D}=7$ heterotic string to be a $K_{3}$ compactified supermembrane. We shall see that it implies a string/membrane duality in $\mathrm{D}=7$. One of the implications of this new duality is that the strongly coupled $T^{3}$-compactified fundamental heterotic

string has a solitonic interpretation as a $K_{3}$ compactified 11-dimensional fivebrane! As confirmation of this prediction, we shall verify that the physical worldsheet field content of the heterotic string is indeed found from the physical field content of the $\mathrm{D}=11$ super-fivebrane by compactification on $K_{3}$.

Conversely, according to $\mathrm{D}=7$ string-membrane duality, the $K_{3}$-compactified fundamental supermembrane has a solitonic interpretation as a $T^{3}$-compactified 10-dimensional fivebrane. This prediction is not satisfied by the relevant fivebrane solutions of $\mathrm{D}=10$ supergravity/YM theory, but this field theory is also not anomay free. It will be argued that the inclusion of the Lorentz Chern-Simons terms needed for anomaly cancellation can lead to the desired result. Finally, we discuss similar issues for the type $\mathrm{D}=10 \mathrm{IIB}$ superstring in the context of a possible generalization of $\mathrm{D}=7$ string/membrane duality to $\mathrm{D}=8$ string/threebrane duality.

\section{String/membrane duality}

Let us start with the $T^{3}$-compactified heterotic string. The effective $\mathrm{D}=7$ field theory, at generic points in the moduli space of this compactification, is $\mathrm{D}=7$ supergravity coupled to 19 vector supermultiplets. The bosonic field content of the supergavity multiplet is [19]

$$
\left(g_{\mu \nu}, B_{\mu \nu}, V_{\mu}^{a}, \sigma\right)
$$

where $V_{\mu}^{a},(a=1,2,3)$, is an $\mathrm{SO}(3)$ triplet of vector potentials (which remain 
abelian in the action of interest to us), and the scalar field $\sigma$ can be identified as the dilaton of string theory. The bosonic field content of the $D=7$ vector multiplet is

$$
\left(V_{\mu}, \phi^{a}\right)
$$

where $\phi^{a}$ is an $\mathrm{SO}(3)$ triplet of scalar fields. The coupling of an arbitrary number, $k$, of vector multiplets to $\mathrm{D}=7$ supergravity has been constructed by Bergshoeff $e t$ al [20]. Since the case of interest here is $k=19$, there are a total of 22 vector fields $V_{\mu}^{I}(I=1, \ldots, 22)$; let us denote by $F_{\mu \nu}^{I}$ their field strength tensors. There are also a total of 58 scalar fields $\left(\sigma, \phi^{i}\right)(i=1 \ldots, 57)$. The 57 scalars $\phi^{i}$ parametrize the sigma-model target space

$$
\mathcal{M}=S O(3,19) /[S O(3) \times S O(19)]
$$

Let us denote by $m_{i j}$ the invariant metric on this coset space. After some field redefinitions the bosonic action of [20] can be rewritten as

$$
S=\int d^{7} x \sqrt{-g} e^{-2 \sigma}\left[R+4(\partial \sigma)^{2}-\frac{1}{3} H^{2}-a_{I J}(\phi) F^{I} F^{J}-2 m_{i j}(\phi) \partial \phi^{i} \partial \phi^{j}\right]
$$

where $a$ is a positive-definite matrix function of the scalars $\phi$ which can be found in [20], and the field strength tensor $H$ of the two-form potential $B$ is given by

$$
H_{\mu \nu \rho}=3 \partial_{[\mu} B_{\nu \rho]}-A_{[\mu}^{I} F_{\nu \rho]}^{J} \eta_{I J}
$$

where $\eta_{I J}$ is the invariant $S O(3,19)$ tensor with diagonal entries $(-1,-1,-1,1, \ldots, 1)$. It follows that the three-form $H$ satisfies the modified Bianchi identity

$$
d H=-\frac{1}{3} F^{I} \wedge F^{J} \eta_{I J}
$$

The action (2.4) has been written in a form appropriate to its interpretation as the effective action of the $\mathrm{D}=7$ heterotic string. If we think of this action 
as obtained by compactification from $\mathrm{D}=10$ on $T^{3}$ then the two-form potential $B$ obviously couples to the fundamental string. It also has a magnetic 'neutral' membrane source obtained by wrapping the $\mathrm{D}=10$ neutral fivebrane around the 3-torus. It is similarly straightforward to determine the $\mathrm{D}=10$ origin of the electric and magnetic sources of the 22 gauge potentials $B_{\mu}^{I}$. Three of these are KaluzaKlein vectors which couple to KK modes and their (magnetic) threebrane duals (obtained by an obvious extension of the standard KK monopole construction, as explained for the $\mathrm{D}=10$ sixbrane in [1]). Three more vector potentials arise from the $\mathrm{D}=10$ two-form potential, and couple to string winding modes and their threebrane duals (the obvious extension of abelian H-monopoles [21,22]). The remaining 16 'heterotic' vectors couple to the charged modes of the additional 16 left-moving wordsheet scalars of the heterotic string, and their magnetic duals, which are presumably (by the available $\mathrm{D}=4$ evidence [23]) threebrane generalizations of BPS monopoles.

The action (2.4) has a dual version in which the two-form potential $B$ is replaced by a three-form $A$ [19]. Since the three-form field strength $H$ has a modified Bianchi identity, the dual version will include a coupling of $A$ to a topological current, as first discussed in [24]. Following the steps presented there, one finds that the dual action can be written as

$$
\begin{aligned}
\tilde{S}=\int d^{7} x\{ & \sqrt{-g} e^{\frac{4}{3} \sigma}\left[R-\frac{8}{3}(\partial \sigma)^{2}-2 m_{i j} \partial \phi^{i} \partial \phi^{j}-\frac{1}{12} F^{2}\right] \\
& \left.-\sqrt{-g} a_{I J} F^{J} F^{J}+\frac{1}{18} \eta_{I J} \varepsilon^{\alpha \beta \gamma \mu \nu \rho \sigma} A_{\alpha \beta \gamma} F_{\mu \nu}^{I} F_{\rho \sigma}^{J}\right\} .
\end{aligned}
$$

This is the effective $\mathrm{D}=7$ action obtained by $K_{3}$ compactification of 11-dimensional supergravity [25]. The last term can be seen to be a direct consequence of the $\varepsilon A F F$ term in 11-dimensions and the fact that $\eta_{I J}$ is the intersection matrix of the 22 linearly independent homology two-cycles of $K_{3}$. What we wish to consider here is the 11-dimensional origin of the various massive modes or extended objects to which the seven dimensional gauge potentials couple. Let us concentrate first on the 22 vector potentials. None of them are KK vectors since $K_{3}$ has no isometries. 
Instead, they all derive from the ansatz

$$
A=B^{I}(x) \wedge \omega_{I}
$$

where the $\omega^{I}$ span the 22-dimensional space of closed but not exact two-forms on $K_{3}$. If we suppose that the 11 dimensional supergravity is merely the effective action for the massless modes of a supermembrane then we must also include the membrane 'wrapping' modes around the 22 two-cycles of $K_{3}$. These are the electrically charged modes that couple to the 22 vector potentials. Their magnetic duals are the 22 threebranes obtained by wrapping the $\mathrm{D}=11$ fivebrane around the same 22 two-cycles. We now turn to the $\mathrm{D}=7$ three-form potential $A$. This is directly descended from the $\mathrm{D}=11$ three-form, so if we suppose that the latter couples to a fundamental 11-dimensional supermembrane then it follows that the $\mathrm{D}=7$ three-form also couples to a fundamental supermembrane. Its magnetic source is the solitonic string obtained by compactification of the $\mathrm{D}=11$ fivebrane on $K_{3}$.

If we dualize the action (2.7) to recast it in the 'heterotic' form of (2.4) then we have a two-form with a solitonic string source and a fundamental magnetic membrane source, i.e. exactly the opposite of what we had before. We therefore see that the dual interpretation of the $\mathrm{D}=7$ Maxwell/Einstein supergravity action either as a toroidally compactified $\mathrm{D}=10$ heterotic string or as a $K_{3}$ compactified $\mathrm{D}=11$ supermembrane leads to a string-membrane duality in $\mathrm{D}=7$. The membrane appears as a soliton in the string theory and the string appears as a soliton in the membrane theory. It seems likely that the string-membrane duality discussed here implies the string-string duality of [26], for which further evidence has recently been found $[4,27,28]$. 


\section{The heterotic string as a compactified $\mathrm{D}=11$ fivebrane}

Perhaps the most interesting aspect of $\mathrm{D}=7$ string/membrane duality is that, from the membrane perspective, the heterotic string theory is really a $K_{3}$ compactification of the $\mathrm{D}=11$ super-fivebrane. It follows that the worldsheet action of the heterotic string should be similarly related to the worldvolume action of the $\mathrm{D}=11$ fivebrane ${ }^{\star}$. Although the latter is unknown, it is known [29] that after (partial) gauge fixing it must be a supersymmetric field theory based on the six-dimensional self-dual $\mathrm{N}=4$ antisymmetric tensor multiplet [30], with field content

$$
\left(S^{[i j]} ; \lambda_{+}^{i} ; A^{+}\right)
$$

Here, $i=(1,2,3,4)$ is an index of $U S p(4) \cong \operatorname{Spin}(5), \lambda_{+}^{i}$ is a chiral 'symplecticMajorana' spinor of the six-dimensional Lorentz group and $A^{+}$is a two-form gauge potential with self-dual three-form field strength. The scalars $S$ form a 5-plet of $\operatorname{Spin}(5)$ and can be interpreted as the Nambu-Goldstone fields due to the breaking of translational invariance in the five transverse directions by the six-dimensional worldsheet of the $\mathrm{D}=11$ fivebrane. In fact, the entire supermultiplet, including the antisymmetric tensor gauge potential, can be given a Nambu-Goldstone interpretation [31]. If we fix the antisymmetric tensor gauge invariance by the choice of lightcone gauge, so reducing the supermultiplet (3.1) to its purely physical field content, we also break the six-dimensional Lorentz group to $S O(1,1) \times[S U(2) \times S U(2)]$. In the compactification of the fivebrane on some four-dimensional space $B$, the $S O(1,1)$ factor is interpreted as the residual wordsheet Lorentz-invariance of the resulting string action, and the $S U(2) \times S U(2)$ factor as the maximal holonomy group of $B$. The worldsheet fields of this effective string action are found as coefficients of the zero modes in the harmonic expansion on $B$ of the physical fields of (3.1). We shall now investigate what these are.

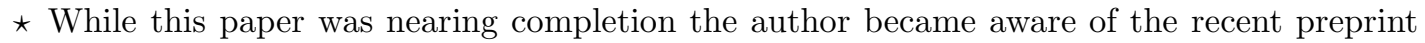
of Harvey and Strominger [28] in which the same idea is developed.
} 
First, the five worldvolume scalars $S^{[i j]}$ produce five worldsheet scalars. These can be identified with the spacetime coordinates $x^{\mu}(\tau, \sigma)$ of the effective $\mathrm{D}=7$ superstring, since wordsheet general coordinate invariance implies that only five of these seven worldsheet fields are physical. Next, we consider the worldvolume spinors $\lambda_{+}^{i}$, which span a vector space of real dimension 16 . Because of their six-dimensional chirality, their $S U(2) \times S U(2)$ representations are correlated with their worldsheet chirality: half of them are $S O(1,1)$ chiral spinors in the $(\mathbf{1}, \mathbf{2})$ representation of $S U(2) \times S U(2)$ while the other half are antichiral $S O(1,1)$ spinors in the $(\mathbf{2}, \mathbf{1})$ representation. The number and chirality of the worldsheet spinors therefore depends on the number and type of covariantly constant spinors admitted by $B$, which depends on the holonomy group of $B$. When $B=T^{4}$, which has a trivial holonomy, we get 8 worldsheet spinors of one chirality and 8 of the other chirality. When $B=K_{3}$, which has $S U(2)$ holonomy, we get only the 8 of one chirality and none of the other chirality. So far, the analysis closely parallels that of [25] for the $K_{3}$ compactification of 11-dimensional supergravity.

Finally, we turn to the two-form potential $A^{+}$, or rather its gauge-invariant field strength three-form $F^{+}=d A^{+}$. The analysis required here closely parallels that given in [32] for the $K_{3}$ compactification of type IIB supergravity: in this case the three-form $F^{+}$yields scalar worldsheet fields $X^{I}$ via the ansatz

$$
F^{+}=d X^{I}(\tau, \sigma) \wedge \omega_{I}
$$

where $\left\{\omega_{I} ; I=1, \ldots, b_{2}\right\}$ span the $b_{2}$-dimensional space of closed but not exact, self-dual or anti-self-dual, two-forms on $B$; i.e. $b_{2}$ is the second Betti number of $B$. Since $F^{+}$is self-dual in six dimensions, the one-forms $d X^{I}$ will be self-dual or anti-self-dual on the string worldsheet according to whether $\omega_{I}$ is self-dual or antiself-dual. This implies that the scalar $X^{I}$ is either left-moving or right moving, i.e. chiral or anti-chiral, according to whether $\omega_{I}$ is self-dual or anti-self-dual. Thus, if $b_{2}=b_{2}^{+}+b_{2}^{-}$, where $b_{2}^{+}$counts the self-dual two-forms and $b_{2}^{-}$counts the anti-selfdual ones, then the two-form potential $A^{+}$on the fivebrane worldsheet will produce 
$b_{2}^{+}$chiral and $b_{2}^{-}$anti-chiral worldsheet scalars. For $B=T^{4}$, we have $b_{2}^{+}=b_{2}^{-}=3$, so the two-form potential $A^{+}$of the $\mathrm{D}=11$ fivebrane yields 3 worldsheet scalars of one chirality and 3 of the other chirality or, equivalently, 3 non-chiral scalars. These can be identified as the maps from the worldsheet to three extra dimensions. Since the total number of physical scalars is 8 , it is clear that the effective string theory is 10 dimensional. Since there are also 8 non-chiral spinors we see that the $T^{4}$ compactified fivebrane has precisely the physical worldsheet field content of the $\mathrm{D}=10$ type IIA string.

For $B=K_{3}$ we have $b_{2}^{+}=3$ and $b_{2}^{-}=19$, so the two-form potential $A^{+}$ of the $\mathrm{D}=11$ fivebrane yields a total of 3 non-chiral worldsheet scalars, which we can give the same interpretation as above, plus 16 additional chiral worldsheet scalars. Thus the physical worldsheet field content of the $K_{3}$ compactified $\mathrm{D}=11$ super-fivebrane is eight non-chiral scalars, 16 additional chiral scalars and eight chiral spinors. This is precisely the physical worldsheet field content of the $\mathrm{D}=10$ heterotic string ${ }^{\star}$.

\section{The supermembrane as a compactified $\mathrm{D}=10$ fivebrane}

We turn now to consider the converse of the prediction of $\mathrm{D}=7$ string-membrane duality that we have just confirmed. This is the prediction that the $K_{3}$-compactified $\mathrm{D}=11$ supermembrane has a solitonic interpretation as a $T^{3}$ compactified fivebrane soliton of the heterotic string. We earlier identified the relevant membrane as the 'neutral' one, since this corresponds to the non-singular membrane solution of the effective $\mathrm{D}=7$ action (2.4). The neutral membrane is a solution of the pure $\mathrm{D}=10$ supergravity theory (and is non-singular in the string metric). As such, the physical field content of the worldvolume action is that of a six-dimensional hypermultiplet, with $4+4$ on-shell boson and fermion degrees of freedom. The worldvolume action was constructed in [33] (where its $K_{3}$ compactification was also briefly discussed).

\footnotetext{
$\star$ Strictly speaking, we have still to show that the spinors have opposite chirality to the 16 additional chiral scalars.
} 
The dimensional reduction of this action on $T^{3}$ is that of the $\mathrm{D}=7$ supermembrane, with no evidence of an 11-dimensional origin. What we need to verify the prediction of string membrane duality is an additional $4+4$ worldsheet boson and fermion degrees of freedom, and their worldsheet action should be that of a supersymmetric sigma model with a $K_{3}$ target space.

To see whether these additional degrees of freedom are present in the relevant $T^{3}$ compactified heterotic fivebrane we must of course consider the full effective $\mathrm{D}=10$ action. This includes the vector supermultiplets of the $E_{8} \times E_{8}$ or $S O(32)$ gauge group, but since they are all zero in the neutral fivebrane solution it would appear that the inclusion of the gauge fields cannot help. However, we should also take into account the fact that the $\mathrm{D}=10$ supergravity/YM theory is not the effective action for the heterotic string because it is anomalous. As Green and Schwarz showed, one must include additional Lorentz Chern-Simons terms in the Bianchi identity for the $\mathrm{D}=10$ three-form field strength, and these must be supersymmetrized. Since the resulting action is an infinite series in powers of the $\mathrm{D}=10$ Riemann tensor, the complete anomaly free and supersymmetric action is unknown, but it is known that the neutral fivebrane requires modification. It seems likely that one must replace the neutral fivebrane by the 'symmetric' fivebrane [17], since this has the same metric and dilaton as the neutral fivebrane solution of $\mathrm{D}=10$ supergravity and it is known to be an exact solution of the classical string theory. Since the YM fields now play a role, it is possible that they or their gaugino superpartners have zero modes, the coefficients of which would constitute additional fields on the fivebrane's worldvolume.

In fact, the YM fields of the symmetric fivebrane are precisely those of the 'gauge' fivebrane solution [34] for which the YM gauge field takes values in an $S O(3)$ subgroup of $E_{8} \times E_{8}$ or $S O(32)$. The zero modes have been discussed in $[34,35]$. Consider the $S O(32)$ case. The adjoint representation of $S O(32)$ has the $S O(3) \times S O(29)$ decomposition

$$
(3,1) \oplus(1,406) \oplus(3,29)
$$


The Atiyah-Singer index theorem implies a zero mode of the gaugino Dirac opeator (in the bosonic background provided by the fivebrane solution) for each $S O(3)$ triplet in this decomposition, of which there are a total of 30. Each of these 30 zero modes yields, on taking into account other bosonic zero modes required by supersymmetry, a worldvolume hypermultiplet. According to this accounting, the physical field content of the fivebrane's worldvolume action is 30 six-dimensional hypermultiplets.

We must now dimensionally reduce on $T^{3}$ to find the worldvolume action of a supermembrane. Here we must take into account that on dimensional reduction a massless six-dimensional hypermultiplet can become a massive three-dimensional hypermultiplet [36], in which case it would not appear in the effective worldvolume action for the supermembrane. To see how this can happen, let $y^{i}$ be the $T^{3}$ coordinates: a massless six-dimensional hypermultiplet coupling to a background $U(1)$ gauge potential $A$ yields a massless three-dimensional hypermultiplet only if the operator $\partial / \partial y^{i}+i A_{i}$ has a zero eigenvalue when acting on functions on $S^{1}$, but for generic constant values of $A_{i}$ this operator has no zero eigenvalues. To apply this observation to the 30 hypermultiplets in the $\mathbf{1} \oplus \mathbf{2 9}$ representation of $S O(29)$, we recall that in compactifying the heterotic string theory on $T^{3}$ we must do so in such a way that the effective seven dimensional field theory is the generic one, i.e. such that $S O(32)$ is spontaneously broken to $U(1)^{16}$. This is done by giving generic expectation values to the components $A_{i}$ of the gauge potentials associated with a maximal abelian subgroup of $S O(32)$. Thus, the only hypermultiplets of the fivebrane's worldvolume action that can be expected to survive as massless hypermultiplets in three dimensions after wrapping the fivebrane around the $T^{3}$ factor of spacetime are those which do not couple to the background gauge fields on $T^{3}$. The 29-plet of hypermultiplets certainly do couple to these fields, so they will not contibute to the three-dimensional worldvolume action.

This leaves only the $S O(29)$ singlet hypermultiplet. It is unclear what happens to it. It couples to the $S O(3)$ gauge potentials but as these are used to construct the solution it is doubtful that they can be considered as 'background' fields. In [34,35] 
this hypermultiplet was identified as the one arising from the partial breaking of translations and supertranslations by the fivebrane solution. If this is right then this $S O(29)$ singlet hypermultiplet certainly remains massless, but then the total number of physical boson and fermion degrees of freedom would be $4+4$, and we would fail to verify the prediction of string-membrane duality. However, it seems odd that worldvolume fields should have both a Nambu-Goldstone and a topological interpretation. After all, the topological argument applies equally (at least for fermion zero modes) to a non-supersymmetric theory, while the argumnt based on symmetry breaking continues to apply in the absence of any YM multiplets. It seems more likely that the singlet worldvolume hypermultiplet of topological origin is an addition to the one of Nambu-Goldstone origin. If it remains massless on $T^{3}$ compactification, as seems possible, there will indeed be a total of $8+8$ boson and fermion three-dimension worldvolume degrees of freedom, as required by stringmembrane duality. It seems a challenging problem to verify this scenario, and to further verify that the action for the 'additional' worldvolume hypermultiplet is that of a supersymmetric three-dimensional sigma model with $K_{3}$ as its target space.

\section{Type IIB and $\mathrm{D}=8$ Dualities}

The type IIB $D=10$ superstring appears to remains aloof from the tangle of relations between the $\mathrm{D}=10$ heterotic and type IIA strings and the $\mathrm{D}=11$ supermembrane ${ }^{\star}$. Nevertheless, the analysis just carried out for the fivebrane solution of $D=11$ supergravity can be repeated for the fivebrane solution of the $D=10$ $\mathrm{N}=2 \mathrm{~B}$ supergravity. In this case the worldvolume action [35] is based on the sixdimensional $\mathrm{N}=4$ vector multiplet

$$
\left(S^{i i^{\prime}} ; \lambda_{+}^{i}, \lambda_{-}^{i^{\prime}} ; V\right)
$$

where $i(=1,2)$ and $i^{\prime}(=1,2)$ are indices of commuting $S U(2)$ groups, i.e. this

\footnotetext{
$\star$ But see [37].
} 
supermultiplet has four scalars, two SU(2)-Majorana spinors of opposite chirality, and a one-form potential $V$. In this case, compactification on $K_{3}$ yields an effective non-chiral string theory with 4 scalars and 4 spinors. This is the field content of a six-dimensional type II superstring, with no evidence of a 10-dimensional origin. Consequently, this is not the field content of an anomaly free string theory. However, while the vector field $V$ does not give any additional worldsheet scalars, it does give a worldsheet vector. A vector has no degrees of freedom in two dimensions but might contribute to the conformal anomaly. Consideration of the $\mathrm{D}=10$ IIB superstring therefore suggests the existence of a six-dimensional type II string theory in which the worldsheet conformal anomaly cancellation is achieved in this way.

Perhaps a better way to bring the type IIB superstring into the picture is by a generalization of $\mathrm{D}=7$ string-membrane duality to $\mathrm{D}=8$ string-threebrane duality. If the $\mathrm{D}=10 \mathrm{~N}=2 \mathrm{~B}$ supergravity is compactified on $T^{2}$ the resulting effective $\mathrm{D}=8$ theory is that of $\mathrm{N}=2 \mathrm{D}=8$ supergravity. It is again true that there are dual forms of the $\mathrm{D}=8$ supergravity action in which one couples naturally to a string and the other to a threebrane, and one can therefore envisage a threebrane theory in which the string appears as a soliton. The obvious candidate for this string soliton is the $\mathrm{D}=10$ self-dual threebrane [3] wrapped around a 2 -torus ${ }^{\dagger}$. The worldvolume action [38] of the self-dual threebrane is based on the four-dimensional $N=4$ vector multiplet

$$
\left(S^{[i j]} ; \lambda_{+}^{i} ; V\right) \quad(i, j=1,2,3,4)
$$

where the scalars $S^{[i j]}$ are a real $S U(4)$ 6-plet, the spinor $\lambda_{+}^{i}$ is a complex chiral $S U(4)$ 4-plet (with anti-chiral complex conjugate in the conjugate $\overline{4}$ irrep. of $S U(4)$ ), and $V$ is an $S U(4)$-singlet one-form potential. Compactification on $T^{2}$ yields the physical worldsheet field content of the type IIA superstring, which is

\footnotetext{
$\dagger$ The $\mathrm{D}=10$ threebrane is geodesically complete and so would qualify as a soliton by the criteria that we have here been insisting on, but, in contrast to the $\mathrm{D}=11$ fivebrane, the multi $\mathrm{D}=10$ threebrane is very probably singular [8].
} 
not unexpected in view of the equivalence between the $T^{2}$ compactified type IIA and type IIB superstrings.

There is also a solitonic threebrane of the $\mathrm{N}=2 \mathrm{D}=8$ supergravity found by wrapping a fivebrane around the two-torus. The threebrane world volume fields of (5.2) are indeed found by $T^{2}$ compactification of either the type IIB or the type IIA fivebrane worldvolume actions. It is interesting to note here that just as the type IIA and IIB superstrings become equivalent on dimensional reduction on $S^{1}$ [39], so also do the worldvolume actions of their respective fivebrane solutions!

The same $D=8$ supergravity that one finds by compactification of the type IIB superstring on $T^{2}$ can also be obtained by compactification of $D=11$ supergravity on $T^{3}$. If the latter is viewed as the effective theory of a fundamental membrane theory then the $D=8$ three-form potential also couples to a fundamental membrane. But the magnetic dual of a membrane in eight dimensions is another membrane. This dual membrane is of course the $T^{3}$ compactification of the $D=11$ fivebrane. An obvious conjecture, analogous to the $D=6$ string-string duality conjecture of $[26,4]$ is a $D=8$ membrane-membrane duality. According to this conjecture, the dual membrane is equivalent to the original one, from which it follows that the $T^{3}$ compactification of the worldvolume action of the $D=11$ fivebrane should yield the worldvolume action of a $T^{3}$ compactified $D=11$ supermebrane. This prediction of $D=8$ membrane-membrane duality is easily checked. Taking into account that a vector potential is equivalent to a scalar in three dimensions, the $T^{3}$ compactification of the $\mathrm{D}=11$ fivebrane's worldvolume action yields an effective three-dimensional action to which the fivebrane's worldvolume spinors contribute $8 S O(2,1)$ Majorana spinors and to which the fivebrane's worldvolume two-form potential $A^{+}$contributes three scalars. Allowing for three-dimensional worldvolume diffeomorphism invariance, the five scalars from the $S$ fields of (3.1) can be promoted to the 8 worldvolume fields that specify the position of a membrane's three-dimensional worldvolume in a $\mathrm{D}=8$ spacetime. Adding to these the 3 scalars from $A^{+}$, we see that the full physical field content is precisely that of the $T^{3}$ compactified $\mathrm{D}=11$ supermembrane, as predicted. 


\section{Comments}

The proposal that the $\mathrm{D}=10$ type IIA superstring theoryis an $S^{1}$-compactified $\mathrm{D}=11$ supermembrane theory leads, when combined with conjectured relations between the heterotic and type IIA superstrings, to the conjecture that the strongly coupled heterotic string in $\mathrm{D}=7$ is equivalent to a $K_{3}$-compactified supermembrane theory. This conjecture has here been shown to imply a string-membrane duality in $\mathrm{D}=7$. One implication of this duality is that from the membrane point of view the $\mathrm{D}=7$ heterotic string is a soliton obtained by $K_{3}$ compactification of the $\mathrm{D}=11$ superfivebrane ${ }^{\ddagger}$. Given this, it follows that the worldsheet action for the heterotic string should be the effective two-dimensional field theory obtained by $K_{3}$ compactification of the six-dimensional worldvolume action of the $\mathrm{D}=11$ superfivebrane. We verified this prediction at the linearized level, i.e. the physical field content. Unfortunately, it is not possible at present to check this prediction beyond the linearized approximation because the full $\mathrm{D}=11$ fivebrane worldvolume action is unknown; its construction is now urgently needed.

Putting together the various p-brane dualities involving the type IIA superstring, one sees that in addition to the heterotic string emerging as a soliton found by a $K_{3}$ compactification of the $\mathrm{D}=11$ fivebrane, the $\mathrm{D}=10$ type IIA superstring has a similar solitonic interpretation as a $T^{4}$ compactified $\mathrm{D}=11$ fivebrane, as does the $\mathrm{D}=11$ supermembrane as a $T^{3}$ compactied $\mathrm{D}=11$ fivebrane. Thus, the worldsheet actions of both the heterotic and the type IIA superstrings, and the worldvolume action of the $\mathrm{D}=11$ supermembrane appear to be effective actions arrived at by compactification of the $\mathrm{D}=11$ fivebrane's worldvolume action. From this perspective, the various string or membrane theories are directly related to the possible choice of compactifying space. It seems hardly possible to avoid the conclusion that the $\mathrm{D}=11$ super-fivebrane must play a fundamental role in string, and membrane, theory; possibly via the $\mathrm{D}=11$ membrane/fivebrane duality suggested in [11]?

$\ddagger$ cf. recent similar results for the $\mathrm{D}=6$ heterotic string $[27,28]$. 


\section{Acknowledgments}

Discussions with M.J. Duff, C.M. Hull, A. Sen and E. Witten are gratefully acknowledged.

\section{REFERENCES}

1. P.K. Townsend, The 11-dimensional supermembrane revisited, preprint hepth-9501068, Phys. Lett. B, in press.

2. E. Bergshoeff, E. Sezgin and P.K. Townsend, Phys. Lett. 189B (1987) 75; Ann. Phys. (N.Y.) 185 (1988) 330.

3. G. Horowitz and A. Strominger, Nucl. Phys. B360 (1991) 197.

4. E. Witten, String theory dynamics in various dimensions, preprint hepth9503124.

5. M.J. Duff, P.S. Howe, T. Inami and K.S. Stelle, Phys. Lett. 191B (1987) 70.

6. M.J. Duff and K.S. Stelle, Phys. Lett. B253 (1991) 113.

7. M.J. Duff, G.W. Gibbons and P.K. Townsend, Phys. Lett. 332 B (1994) 321.

8. G.W. Gibbons, G.T. Horowitz and P.K. Townsend, Class. Quantum Grav. 12 (1995) 297.

9. A. Dabholkar, G.W. Gibbons, J.A. Harvey and F. Ruiz-Ruiz, Nucl. Phys. B340 (1990) 33.

10. R. Güven, Phys. Lett. 276B (1992) 49.

11. C.M. Hull and P.K. Townsend, Nucl. Phys. B438 (1995) 109.

12. I. Bars, C.N. Pope and E. Sezgin, Phys. Lett. 198B (1987) 455.

13. C.N. Pope and K.S. Stelle, Class. Quantum Grav. 5 (1988) L161. 
14. B. de Wit and H. Nicolai, Supermembranes - a fond farewell? in Supermembranes and Physics in 2+1 Dimensions, eds. M.J. Duff, C.N. Pope and E. Sezgin (World Scientific, 1990).

15. C.M. Hull and P.K. Townsend, in preparation.

16. M.J. Duff and J.X. Lu, Nucl. Phys. B354 (1991) 141.

17. C. Callan, J.A. Harvey and A. Strominger, Nucl. Phys. B359 (1991) 611.

18. A. Dabholkar and J.A. Harvey, Phys. Rev. Lett. 63 (1989) 719.

19. P.K. Townsend and P. van Nieuwenhuizen, Phys. Lett. 125B (1983) 41; L. Mezincescu, P.K. Townsend and P. van Nieuwenhuizen, Phys. Lett. 143B (1984) 384.

20. E. Bergshoeff, I.G. Koh and E. Sezgin, Phys. Rev. D32 (1985) 1353.

21. R.R. Khuri, Phys. Lett. B259 (1991) 261; Nucl. Phys. B387 (1992) 315.

22. J. Gauntlett, J. Harvey and J. Liu, Nucl. Phys. B409 (1993) 363.

23. A. Sen, Int. J. Mod. Phys. A8 (1993) 5079.

24. H. Nicolai and P.K. Townsend, Phys. Lett. 98B (1981) 257.

25. M.J. Duff, B.E.W. Nilsson and C.N. Pope, Phys. Lett 129B (1983) 39.

26. M.J. Duff, Strong/Weak Coupling Duality From the Dual String, preprint hep-th/9501030.

27. A. Sen, String-String Duality Conjecture in Six Dimensions and Charged Solitonic Strings, preprint hep-th/9504027.

28. J.A. Harvey and A. Strominger, The Heterotic String is a Soliton, preprint hep-th/9504047.

29. G.W. Gibbons and P.K. Townsend, Phys. Rev. Lett. 71 (1993) 3754.

30. P.S. Howe, G. Sierra and P.K. Townsend, Nucl. Phys. B221 (1983) 331.

31. P.K. Townsend, Phys. Lett. 309B (1993) 33. 
32. P.K. Townsend, Phys. Lett. 139B (1984) 283.

33. A. Achúcarro, J. Evans, P.K. Townsend and D. Wiltshire, Phys. Lett. 198B (1987) 441.

34. A. Strominger, Nucl. Phys. B343 (1990) 167.

35. C. Callan, J.A. Harvey and A. Strominger, Nucl. Phys. B367 (1991) 60.

36. G. Sierra and P.K. Townsend, Nucl. Phys. B223 (1984) 289.

37. S.J. Gates, Jr. and V.G.J. Rodgers, A Truly Crazy Idea about Type IIB Supergravity and Heterotic Sigma-Models, preprint hep-th/9503237.

38. M.J. Duff and J.X. Lu, Phys. Lett. 273B (1991) 409.

39. J. Dai, R.G. Leigh and J. Polchinski, Mod. Phys. Lett. A4 (1989) 2073; M. Dine, P. Huet and N. Seiberg, Nucl. Phys. B322 (1989) 301. 